Ciência Florestal, Santa Maria, v. 24, n. 1, p. 193-204, jan.-mar., 2014

ISSN 0103-9954

\title{
EFEITO DO DESBASTE NAS RACHADURAS DE TOPO DAS TORAS DE Eucalyptus grandis W. Hill ex Maiden
}

\author{
EFFECT OF THINNING IN THE END SPLITS OF THE LOGS OF Eucalyptus grandis W. Hill ex \\ Maiden
}

\author{
Rômulo Trevisan ${ }^{1}$ Clovis Roberto Haselein ${ }^{2}$ Elio José Santini ${ }^{3}$ Luciano Denardi ${ }^{4}$ \\ Darci Alberto Gatto 5
}

\begin{abstract}
RESUMO
O estudo teve como objetivo avaliar o efeito do desbaste nas rachaduras de topo das toras de Eucalyptus grandis aos 18 anos de idade. Para tanto, foi analisado um experimento situado próximo ao litoral do Rio Grande do Sul, instalado em delineamento blocos ao acaso com quatro repetições. Os sete tratamentos foram definidos devido ao número de desbastes aplicados e variaram de zero até seis intervenções. As árvores foram selecionadas, em cada tratamento, com base no diâmetro dominante de Assmann e no diâmetro da árvore central, sendo denominadas de árvores dominantes e centrais, respectivamente. Após o abate foram selecionadas as toras localizadas entre as posições do DAP (diâmetro a altura do peito) e 25\%; $25 \%$ e $50 \% ; 50 \%$ e $75 \% ; 75 \%$ e $100 \%$ da altura comercial para o estudo das rachaduras de topo. Os resultados indicaram que o índice médio de rachadura de topo das árvores dominantes e centrais foi maior na segunda tora amostrada, evidenciando que a toragem das árvores deve ser realizada em conjunto com técnicas que minimizem as tensões de crescimento, principalmente quando o seccionamento for realizado próximo à metade da altura comercial. O desbaste induziu a variação do índice médio de rachadura de topo das toras, tanto nas árvores dominantes quanto nas centrais; porém, não foi verificada uma tendência clara positiva ou negativa, podendo-se concluir que alterações na taxa de crescimento não afetaram esse parâmetro de maneira uniforme.
\end{abstract}

Palavras-chave: eucalipto; tratos silviculturais; qualidade da madeira.

\begin{abstract}
The study was undertaken to evaluate the effect of thinning in the end splits of the logs of the Eucalyptus grandis at 18 years of age. There was analyzed a thinning experiment, located in the northern coast of Rio Grande do Sul State, Brazil, installed in randomized blocks with four repetitions. The seven treatments were defined according to the number of thinnings applied and ranged from zero to six interventions. The trees were selected, in each treatment, on the basis of the Assmann's dominant diameter and the central diameter of tree, being referred to as dominant and central trees, respectively. After cutting down the selected the logs were located between the DBH (diameter at breast height) and $25 \%$ positions, $25 \%$ and $50 \%$; $50 \%$ and

1 Engenheiro Florestal, Dr., Professor Adjunto do Departamento de Engenharia Florestal, Centro de Educação Superior Norte do RS, Universidade Federal de Santa Maria, BR 386 Km 40, Linha 7 de Setembro, CEP 98400-000, Frederico Westphalen (RS), Brasil. romulo_trevisan@ufsm.br

2 Engenheiro Florestal, PhD., Professor Associado do Departamento de Ciências Florestais, Centro de Ciências Rurais, Universidade Federal de Santa Maria, Av. Roraima, 1000, CEP 97105-900, Santa Maria (RS), Brasil. clovis.haselein@ufsm.br

3 Engenheiro Florestal, Dr., Professor Associado do Departamento de Ciências Florestais, Centro de Ciências Rurais, Universidade Federal de Santa Maria, Av. Roraima, 1000, CEP 97105-900, Santa Maria (RS), Brasil. santini@ufsm.br

4 Engenheiro Florestal, Dr., Professor Adjunto do Departamento de Engenharia Florestal, Centro de Educação Superior Norte do RS, Universidade Federal de Santa Maria, BR 386 Km 40, Linha 7 de Setembro, CEP 98400-000, Frederico Westphalen (RS), Brasil. lucianodenardi@yahoo.com.br

5 Engenheiro Florestal, Dr., Professor Adjunto do Curso de Engenharia Industrial Madeireira, Departamento de Engenharia Agrícola, Universidade Federal de Pelotas, Caixa Postal 354, CEP 96019-900, Pelotas (RS), Brasil. darcigatto@yahoo.com

Recebido para publicação em 24/01/2011 e aceito em 27/03/2013
\end{abstract}


$75 \% ; 75 \%$ and $100 \%$ of the commercial height, for the end split study. The results indicate that the average end splits of the logs in the dominant and central trees was higher at the second log sampled, suggesting that sectioning should be carried in conjunction with techniques that minimize the growth stress, especially when this is performed near the half of the commercial height. The thinning induced variation in the logs end split, both for dominant and central trees; however, there was no clear positive or negative trend, leading to the conclusion that changes in growth rate did not affect these parameters uniformly.

Keywords: Eucalyptus; silvicultural treatments; wood quality.

\section{INTRODUÇÃO}

As restrições impostas à utilização de madeiras provenientes de espécies nativas euxilóforas, devido ao uso insustentável dessa matéria-prima e à crescente preocupação com a conservação de nossas florestas, foram quesitos importantes para o êxito do gênero Eucalyptus no setor florestal brasileiro. Desde então, a busca por produtividade e qualidade desses povoamentos florestais tem sido alvo de inúmeras pesquisas (TREVISAN et al., 2008).

Conforme Del Menezzi (1999), Assis (2000) e Calonego e Severo (2005), o gênero Eucalyptus é, por vezes, referido como de difícil processamento, em razão dos altos níveis de tensões de crescimento, os quais, aliados aos defeitos decorrentes da secagem, dificultam a obtenção de material com qualidade superior. As rachaduras associadas a essas tensões, juntamente com os defeitos de secagem, trazem como resultado perdas significativas de material durante o processamento industrial, aspectos considerados como principais entraves à utilização econômica desse gênero (MALAN, 1979; JANKOWSKY, 1995; HASELEIN et al., 2004; TRUGILHO et al., 2004; GONÇALEZ et al., 2006; LIMA et al., 2007).

De acordo com Latorraca e Albuquerque (2000), Trugilho et al. (2004) e Calonego e Severo (2005), o impacto das tensões de crescimento sobre a madeira resulta no aparecimento de rachaduras de topo e rachaduras internas, no momento em que a pressão (tensão) é liberada, ou seja, no momento do corte. Assim, conforme Malan (1979) e Garcia (1995), a zona periférica da tora, sob tração, tende, após a derrubada, a contrair-se e, a parte central sob compressão, a expandir-se, causando as rachaduras de topo nas toras.

Oliveira et al. (1999) e Gonçalez et al. (2006) relatam que árvores com elevado nível de tensões de crescimento desenvolvem rachaduras radiais durante e após o abate, principalmente se tais árvores forem mantidas diretamente expostas ao sol. Essas rachaduras de topo normalmente ocorrem até um período de 3 dias (MALAN, 1979);
4 dias (SCHACHT e GARCIA, 1997; SCHACHT et al., 1998); 5 dias (BARISKA, 1990) ou uma semana após a derrubada (OLIVEIRA et al., 1999). Posteriormente a esse período, geralmente ocorrem fendilhamentos adicionais, além de empenamentos nas tábuas, devido à influência de outros tipos de tensões existentes nas toras (HILLIS, 1968; PURNELL, 1988).

Atualmente existem poucas informações a respeito da influência da intervenção de desbaste nas tensões de crescimento. Entretanto, Nutto e Maestri (2002) evidenciaram que o nível e a distribuição das tensões de crescimento em árvores dependem, provavelmente, de parâmetros de crescimento que podem ser influenciados por meio do manejo.

Conforme Ferrand (1983) e Wilkins e Kitahara (1991), intervenções silviculturais de desbaste efetuadas em uma população de Eucalyptus grandis, aos 12,5 anos de idade, aumentaram a taxa de crescimento das árvores e diminuíram a tensão de crescimento. Conclusões semelhantes foram descritas por Miranda e Nahuz (1999), que afirmam que o maior espaçamento de plantio reduziu a percentagem de rachaduras de topo das tábuas e, consequentemente, a perda de material serrado.

Contudo, Malan (1988) e Lima et al. (2007), em seus relatos sobre a espécie Eucalyptus grandis, supõem que as práticas silviculturais não podem ser caracterizadas como um instrumento único para reduzir o nível de tensões de crescimento, pois os fatores genéticos também podem estar envolvidos.

No que se refere à variação da tensão de crescimento com a altura da árvore, a bibliografia revela que existem 4 tipos de tendências: sem correlação (KUBLER, 1987; SANTOS, 2002; LIMA et al., 2007); crescente (CHAFE, 1981; GARCIA, 1995; MIRANDA e NAHUZ, 1999; TRUGILHO et al., 2002); crescente até a metade da altura da árvore e a partir desse ponto decrescente (PURNELL, 1988; MELLADO, 1993; DEL MENEZZI, 1999; LIMA et al., 2000;); e decrescente (CHAFE, 1985; SCHACHT e GARCIA, 1997).

Dessa forma, o presente estudo teve por objetivo verificar o efeito do desbaste nas rachaduras 
de topo das toras de Eucalyptus grandis W. Hill ex Maiden, aos 18 anos de idade, bem como a variação longitudinal desse fenômeno em árvores dominantes e centrais, no intuito de fornecer mais informações relacionadas à qualidade do material quando submetido a essa intervenção.

\section{MATERIAL E MÉTODO}

\section{Características do experimento}

O material utilizado no estudo foi proveniente de um povoamento experimental pertencente à Empresa Flosul Indústria e Comércio de Madeiras Ltda., localizada no município de Capivari do Sul, litoral norte do estado do Rio Grande do Sul. O experimento situa-se aproximadamente nas coordenadas geográficas de $50^{\circ} 30^{\prime}$ de longitude oeste e $30^{\circ} 08^{\prime}$ de latitude sul.

O povoamento experimental foi implantado em maio de 1990 com espaçamento inicial de $3,0 \times 1,7 \mathrm{~m}$, utilizando uma tentativa de monitoramento do crescimento por Curvas de Tendências Correlacionadas (Correlated Curve Trend - CCT), inicialmente formulada por O'Conner para pesquisas com Pinus, na África do Sul (REINSTORF, 1970). Entretanto, o típico experimento CCT, no qual as áreas das parcelas podem variar de $4046,84 \mathrm{~m}^{2}$ a $809,36 \mathrm{~m}^{2}$, foi modificado por Bredenkamp (1984). O novo enfoque definiu o tamanho das parcelas, fixando em 49 o número de árvores que deve existir no interior das parcelas desbastadas, incluindo as de bordadura e 25 árvores úteis. Esse procedimento, denominado de Amostra de Tamanho Padronizado (Standardize the Sample Size - SSS), além de permitir a redução ou o aumento do número de parcelas com tratamentos de acordo com a variação de espaço vital buscada nos desbastes, assegurou maior confiabilidade e igual probabilidade de confiança, ao contrário do método original.

O delineamento utilizado para executar o programa de desbaste pelo método SSS-CCT foi o em blocos ao acaso, com quatro repetições para cada um dos 7 tratamentos experimentais analisados. No experimento, foram executadas de zero até seis intervenções de desbaste por baixo, de acordo com o plano de redução do número de árvores por hectare, ou seja, em cada tratamento foram retiradas diferentes quantidades de árvores dominadas e com dimensões inferiores, favorecendo o desenvolvimento das árvores de maiores dimensões. A Tabela 1 apresenta os tratamentos empregados para verificar a variação dos parâmetros a serem estudados em relação à intervenção de desbaste, aos 18 anos de idade.

O primeiro desbaste, com redução de 1960 para 1176 árvores por hectare, foi realizado antes que o povoamento manifestasse qualquer sinal de competição, sendo executado nos tratamentos 2 a 7, aos quatro anos de idade. É interessante observar que o tratamento 1 permaneceu inalterado, sem nenhum desbaste e contendo a população original (Tabela 1).

Aos cinco anos de idade foirealizado o segundo desbaste, com redução de 1176 para 784 árvores por hectare, nos tratamentos 3 a 7. Cabe ressaltar que o planejamento dos desbastes era anual, porém,

TABELA 1: Tratamentos de desbaste analisados aos 18 anos de idade.

TABLE 1: Thinning treatments analyzed at 18 years old.

\begin{tabular}{|c|c|c|c|c|c|c|c|c|c|}
\hline \multirow{3}{*}{ Trat. } & \multirow{2}{*}{$\begin{array}{c}\text { Implantação } \\
\text { (1990) }\end{array}$} & \multicolumn{6}{|c|}{ Número de desbastes aplicados } & \multirow{3}{*}{$\begin{array}{c}\begin{array}{c}\text { Rotação } \\
(2008)\end{array} \\
\begin{array}{c}18 \text { anos } \\
(\mathrm{N})\end{array}\end{array}$} & \multirow{3}{*}{$\begin{array}{c}\mathrm{G} \\
\left(\mathrm{m}^{2} / \mathrm{ha}\right)\end{array}$} \\
\hline & & 1 & 2 & 3 & 4 & 5 & 6 & & \\
\hline & $\begin{array}{c}0 \text { ano } \\
(\mathrm{N})\end{array}$ & $\begin{array}{l}4 \text { anos } \\
(\mathrm{N})\end{array}$ & $\begin{array}{c}5 \text { anos } \\
(\mathrm{N})\end{array}$ & $\begin{array}{c}10 \text { anos } \\
(\mathrm{N})\end{array}$ & $\begin{array}{l}11 \text { anos } \\
(\mathrm{N})\end{array}$ & $\begin{array}{c}12 \text { anos } \\
(\mathrm{N})\end{array}$ & $\begin{array}{l}13 \text { anos } \\
(\mathrm{N})\end{array}$ & & \\
\hline 1 & 1960 & & & & & & & 1960 & 53,5 \\
\hline 2 & 1960 & 1176 & & & & & & 1176 & 51,4 \\
\hline 3 & 1960 & 1176 & 784 & & & & & 784 & 47,1 \\
\hline 4 & 1960 & 1176 & 784 & 588 & & & & 588 & 40,9 \\
\hline 5 & 1960 & 1176 & 784 & 588 & 392 & & & 392 & 34,6 \\
\hline 6 & 1960 & 1176 & 784 & 588 & 392 & 294 & & 294 & 25,6 \\
\hline 7 & 1960 & 1176 & 784 & 588 & 392 & 294 & 196 & 196 & 20,7 \\
\hline
\end{tabular}

Em que: Trat. = tratamentos de desbaste; $\mathrm{N}=$ número de árvores por hectare; $\mathrm{G}=$ área basal por hectare, $\mathrm{m}^{2} / \mathrm{ha}$. 
devido a problemas técnicos enfrentados pela empresa, o terceiro desbaste foi executado somente cinco anos depois, quando o povoamento tinha dez anos de idade, nos tratamentos 4 a 7 , com redução de 784 para 588 indivíduos por hectare.

O quarto desbaste foi realizado no ano seguinte, nos tratamentos 5 a 7 , e reduziu de 588 para 392 árvores por hectare. Aos doze anos de idade, foi executado o quinto desbaste, nos tratamentos 6 e 7, com redução de 392 para 294 árvores por hectare. Por fim, o último desbaste foi aplicado aos treze anos de idade e reduziu de 294 para 196 o número de indivíduos por hectare no tratamento 7 .

A seleção das árvores foi realizada com base no DAP, tendo sido retiradas, de cada tratamento e de cada bloco, uma árvore dominante e uma árvore central, totalizando 56 árvores. Para a escolha da árvore dominante, foi empregado o conceito de Assmann, no qual, em um hectare, as 100 árvores de maior diâmetro são mensuradas. Utilizando regra de três simples e considerando a área interna de cada parcela, foi determinado o número de árvores dominantes e, em seguida, calculada a árvore dominante média a ser amostrada $\left(\mathrm{d}_{100}\right)$. Para a seleção da árvore central $\left(\mathrm{d}_{\mathrm{z}}\right)$, procedeu-se da forma tradicional, eliminando o menor e o maior indivíduo, alternadamente, até chegar à $\mathrm{d}_{\mathrm{z}}$. No caso de restarem duas árvores ao final, a escolha foi feita por sorteio (TREVISAN, 2010).

\section{Rachaduras de topo das toras}

Para a análise das rachaduras de topo das árvores dominantes e das centrais, selecionadas em cada tratamento, foram confeccionadas quatro toras formadas entre as posições do DAP e $25 \% ; 25 \%$ e $50 \%$; $50 \%$ e $75 \%$; e $75 \%$ e $100 \%$ da altura comercial, definida pelo diâmetro de $13 \mathrm{~cm}$, que corresponde ao limite para entrada de toras na serraria da empresa, totalizando 224 unidades amostradas.

O comprimento total de cada tora, igual a 3,30 $\mathrm{m}$ a partir das posições iniciais já mencionadas, também seguiu as especificações de desdobro da empresa. Por essa razão e devido à coleta de material para outras análises, a primeira porção da árvore formada pela base $(0,10 \mathrm{~m})$ e DAP não foi amostrada. Cabe ressaltar ainda que, no momento da toragem, as árvores foram devidamente apoiadas para evitar as rachaduras resultantes das tensões de flexão.

Após o dimensionamento, cada tora recebeu, em ambas as extremidades, uma numeração de acordo com sua localização experimental e posicionamento em relação à altura, para em seguida, ser coberta com saco plástico. Essa ação teve por objetivo minimizar as perdas naturais de umidade e, consequentemente, os efeitos da secagem ao ar livre no desenvolvimento de rachaduras.

As toras permaneceram no campo sem sofrer qualquer restrição mecânica por um período de quatro dias após o abate. No início do quinto dia, o saco plástico foi retirado de cada extremidade da amostra, e as mensurações de abertura máxima e comprimento das rachaduras na seção transversal (base e topo), quando ocorriam, assim como o raio médio livre desse defeito, foram realizadas com auxílio de um paquímetro digital com precisão de $0,01 \mathrm{~mm}$. É interessante destacar que o período de permanência das toras no campo foi baseado na literatura mencionada por Hillis (1968) e Purnell (1988), a qual relata a influência da tensão de crescimento no desenvolvimento de rachaduras de topo das toras até um limite máximo de 7 dias após a derrubada das árvores.

Os dados coletados foram empregados no cálculo do índice de rachadura, utilizando metodologia proposta por Lima (2000), que consiste na mensuração direta de cada rachadura de topo de tora, sem atribuição de pesos, expresso por:

$$
\operatorname{IRT}=200\left(\frac{\sum_{i=1}^{n} a_{i} C_{i}}{\pi D^{2}}\right)
$$

Em que: IRT = índice de rachadura de topo de tora das árvores dominantes e centrais, \%; D = diâmetro médio da seção de corte, $\mathrm{cm} ; \mathrm{a}_{\mathrm{i}}=$ abertura máxima da rachadura $(\mathrm{i}=1 ; \ldots ; \mathrm{n}), \mathrm{cm} ; \mathrm{C}_{\mathrm{i}}=$ comprimento da rachadura, $\mathrm{cm}$.

\section{Análise estatística dos dados}

A análise estatística do efeito das diferentes intensidades de desbaste no desenvolvimento de rachaduras de topo foi realizada após a determinação da posição relativa média no sentido axial (base-topo), correspondente à média dos valores em percentual de cada extremidade da tora e do índice médio de rachaduras de topo das toras, conforme descrito anteriormente.

Dessa forma, para o estudo da influência do espaço vital no índice médio de rachaduras de topo das toras das árvores dominantes e centrais e da respectiva variação no sentido axial, os dados foram submetidos à análise de regressão pelo procedimen- 
to Stepwise de modelagem de regressão utilizando o pacote "Statistical Analysis System" (SAS, 1993). O modelo gigante foi definido por:

$$
\operatorname{IMRT} ; \ln (\mathrm{IMRT})=\mathrm{f}\left(\mathrm{P} ; \frac{1}{\mathrm{P}} ; \mathrm{P}^{2} ; \frac{1}{\mathrm{P}^{2}} ; \ln \mathrm{P} ; \frac{1}{\ln \mathrm{P}} ; \sqrt{\mathrm{P}} ; \frac{1}{\sqrt{\mathrm{P}}}\right)
$$

Em que: IMRT = índice médio de rachadura de topo de tora das árvores dominantes e centrais, $\% ; \mathrm{P}=$ posição relativa média (sentido base-topo), $\%$.

O melhor modelo foi selecionado com base no coeficiente de determinação ajustado $\left(\mathrm{R}_{\mathrm{aj}}^{2}\right)$, erro padrão da estimativa $\left(\mathrm{S}_{\mathrm{xy}}\right)$, valor de $\mathrm{F}$ calculado e análise da distribuição dos resíduos. Após esse procedimento, no modelo escolhido para estimar a variação axial desse defeito, no estrato dominante e médio, foram adicionadas variáveis Dummy, que assumiram valores de 0 e 1 , conforme o tratamento de desbaste aplicado em cada parcela, da seguinte forma:

$\mathrm{Di}=1$, se a árvore estivesse presente na parcela/tratamento com desbaste $\mathrm{i}$;

$\mathrm{Di}=0$, se a árvore estivesse ausente nessa parcela/tratamento com desbaste i;

Com isso, foi possível expressar as regressões individuais ajustadas para os sete tratamentos de desbaste em função de uma regressão linear múltipla, denominada de modelo gigante, representada pelas variáveis independentes descritas na equação a seguir:

\section{IMRT $=f(X ; D i ; D i . X)$}

Em que: IMRT $=$ índice médio de rachadura de topo de tora das árvores dominantes e centrais, $\% ; \mathrm{X}=$ posição relativa média (sentido base-topo) selecionada pelo procedimento Stepwise de regressão, \%; Di = Dummy (tratamento de desbaste, onde $\mathrm{i}=1 ; 2 ; 3 ; 4 ; 5 ; 6$ e 7); Di.X = interação da variável Di com a variável $\mathrm{X}$.

Considerando a possibilidade dos dados de rachadura de topo de tora das árvores dominantes e centrais apresentarem uma tendência semelhante de variação axial e assim agrupar os estratos amostrados para verificar a influência dos desbastes, foi aplicado o Teste-t para duas amostras com variância equivalentes. As hipóteses verificadas foram h0: variação axial homogênea entre estratos (todos os procedimentos anteriores novamente realizados, porém, com os dados agrupados) e h1: variação axial heterogênea entre estratos. Esse procedimento foi executado após os dados terem sido submetidos ao Teste de Bartlett de homogeneidade de variâncias.

\section{RESULTADOS E DISCUSSÃO}

\section{Árvores dominantes}

A equação selecionada para estimar a variação do índice médio de rachadura de topo de tora no sentido axial das árvores dominantes, com coeficiente de variação de $42 \%$ e com erro padrão da estimativa igual a $0,64 \%$, foi expressa por:

$$
\operatorname{IMRT}=\mathrm{e}^{1,784440-0,000262 \mathrm{P}^{2}-4,312720(1 / \sqrt{\mathrm{P}})}
$$

Em que: IMRT = índice médio de rachadura de topo de tora, $\% ; \mathrm{P}=$ posição relativa média no sentido base-topo, $\%$.

Determinado o padrão de variação axial desse índice, os dados foram então relacionados com os respectivos tratamentos de desbaste e submetidos à análise de regressão com o emprego de variáveis Dummy, conforme mostra a Tabela 2.

Os resultados encontrados indicam que a equação selecionada pelo procedimento Stepwise de modelagem de regressão, para estimar a variação longitudinal do índice médio de rachadura de topo de tora, não pode ser utilizada para todas as intensidades de desbaste analisadas, pois a Dummy correspondente ao tratamento 3 (D3), utilizada no modelo gigante, foi significativa em nível de $5 \%$ de probabilidade de erro. Cabe ressaltar que nenhuma das interações entre o desbaste e a posição relativa média no sentido axial foi significativa ao mesmo nível de probabilidade (Tabela 2).

Dessa forma, com vistas a analisar as diferenças do índice médio de rachadura de topo de tora entre os desbastes, detectadas na análise de variância, os dados foram plotados em função da posição relativa média no sentido base-topo, ajustando-se à equação selecionada pelo procedimento Stepwise. A Figura 1 mostra os valores desse índice para a espécie Eucalyptus grandis, estimados pelo modelo gigante abaixo descrito, nas sete intervenções silviculturais de desbaste estudadas.

$$
\text { IMRT }=\mathrm{e}^{1,82117-0,00026 \mathrm{P}^{2}-4,32961(1 / \sqrt{\mathrm{P}})-0,23331 \mathrm{D} 3}
$$

Em que: IMRT = índice médio de rachadura de topo de tora, \%; $\mathrm{P}=$ posição relativa média no sentido base-topo, \%; D3 = variável Dummy correspondente ao tratamento 3 (dois desbastes executados).

De acordo com a Figura 1, o índice médio de rachadura de topo de tora, nas árvores dominantes da madeira de Eucalyptus grandis, apresentou 
TABELA 2: Análise de variância da regressão com variável Dummy (tipo SS1) do índice médio de rachadura de topo de tora das árvores dominantes de Eucalyptus grandis em função da posição relativa média e do tratamento de desbaste.

TABLE 2: Analysis of variance of regression with Dummy variable (SS1 type) of average end split index of logs of Eucalyptus grandis dominant trees in function of the average relative position and thinning treatment.

\begin{tabular}{cccccc}
\hline FV & GL & SQ & QM & F & Prob. $>$ F \\
\hline Modelo & 14 & 18,23415 & 1,302439 & 8,46 & $<, 0001^{* *}$ \\
$\mathrm{P}^{2}$ & 1 & 11,53700 & 11,53700 & 74,92 & $<, 0001^{* *}$ \\
$1 /(\mathrm{P})^{1 / 2}$ & 1 & 5,141229 & 5,141229 & 33,39 & $<, 0001^{* *}$ \\
D1 & 1 & 0,050299 & 0,050299 & 0,33 & $0,5692^{\text {ns }}$ \\
D2 & 1 & 0,195025 & 0,195025 & 1,27 & $0,2638^{\text {ns }}$ \\
D3 & 1 & 0,721260 & 0,721260 & 4,68 & $0,0334^{*}$ \\
D4 & 1 & 0,008201 & 0,008201 & 0,05 & $0,8181^{\text {ns }}$ \\
D5 & 1 & 0,120235 & 0,120235 & 0,78 & $0,3795^{\text {ns }}$ \\
D6 & 1 & 0,104803 & 0,104803 & 0,68 & $0,4118^{\text {ns }}$ \\
D7 & 0 & 0 & 0 & 0 & 0 \\
D1.P & 1 & 0,002424 & 0,002424 & 0,02 & $0,9005^{\text {ns }}$ \\
D2.P & 1 & 0,114229 & 0,114229 & 0,74 & $0,3916^{\text {ns }}$ \\
D3.P & 1 & 0,006091 & 0,006091 & 0,04 & $0,8428^{\text {ns }}$ \\
D4.P & 1 & 0,000875 & 0,000875 & 0,01 & $0,9401^{\text {ns }}$ \\
D5.P & 1 & 0,213743 & 0,213743 & 1,39 & $0,2422^{\text {ns }}$ \\
D6.P & 1 & 0,018729 & 0,018729 & 0,12 & $0,7282^{\text {ns }}$ \\
D7.P & 0 & 0 & 0 & 0 & 0 \\
Erro & 81 & 12,473688 & 0,153996 & - & - \\
Total & 95 & 30,707837 & - & - & - \\
\hline
\end{tabular}

Em que: $\mathrm{FV}$ = fonte de variação; $\mathrm{GL}=$ graus de liberdade; $\mathrm{SQ}=$ soma dos quadrados; $\mathrm{QM}=$ quadrado médio; $\mathrm{F}$ = valor de F calculado; Prob. $>\mathrm{F}$ = nível de probabilidade de erro; $\mathrm{P}=$ posição relativa média no sentido base-topo, $\%$; $\mathrm{D}_{\ldots, \mathrm{i}}=$ tratamentos de desbaste (Dummy); $\mathrm{D}_{\ldots, \mathrm{p}} \mathrm{P}^{2}=$ interação tratamento (Dummy) com a variável $\mathrm{P}^{2} ; * *=$ significativo ao nível de $1 \%$ de probabilidade de erro; $*$ = significativo ao nível de $5 \%$ de probabilidade de erro; ns = não significativo ao nível de $5 \%$ de probabilidade de erro.

o mesmo padrão de variação no sentido base-topo para todas as intensidades de desbaste analisadas. Observa-se que os valores desse parâmetro têm acréscimo da base até, aproximadamente, $30 \%$ da altura comercial e, a partir dessa posição, um decréscimo com a altura. Os resultados encontrados estão de acordo com o modelo de variação longitudinal descrito por Purnell (1988), Mellado (1993), Del Menezzi (1999) e Lima (2000).

Com relação ao desbaste, verifica-se, na Figura 1, que houve influência das diferentes formas de competição proporcionadas pelas diferentes intensidades de desbaste no índice médio de rachadura de topo de tora. Entretanto, esse fato justifica-se apenas pela variação encontrada no tratamento 3 (2 desbastes executados), o qual apresentou um índice menor em relação aos demais tratamentos estudados.

\section{Árvores centrais}

A equação de regressão selecionada para estimar a variação axial do índice médio de rachadura de topo de tora nas árvores centrais, com coeficiente de variação igual a $50 \%$ e erro padrão da estimativa de $0,59 \%$, foi expressa por:

$$
\operatorname{IMRT}=\mathrm{e}^{0,221780-0,000063 \mathrm{P}^{2}}
$$

Em que: IMRT = índice médio de rachadura de topo de tora, $\% ; \mathrm{P}=$ posição relativa média no sentido base-topo, $\%$. 

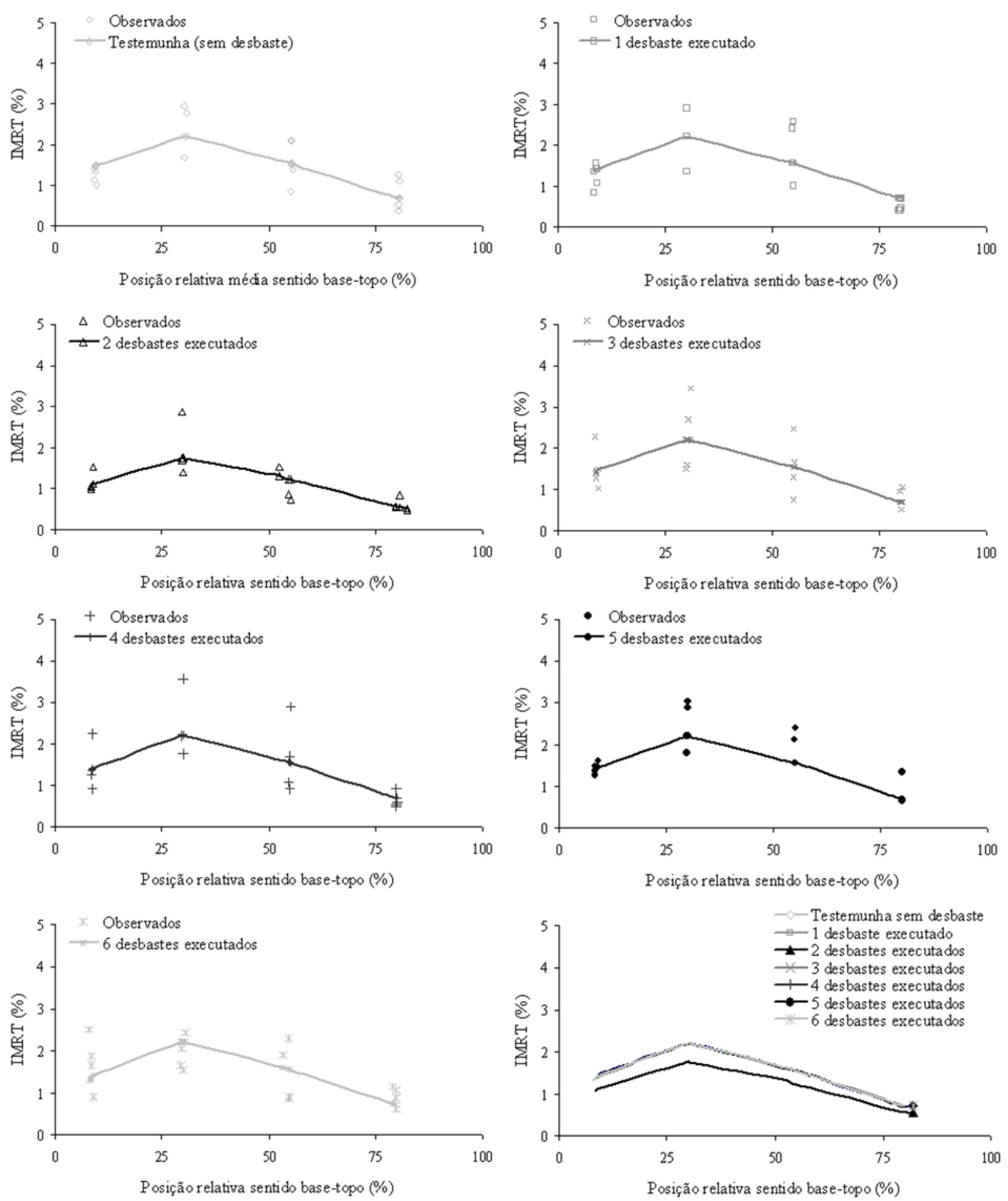

FIGURA 1: Variação do índice médio de rachadura de topo de tora (IMRT) das árvores dominantes de Eucalyptus grandis em função da posição relativa média no sentido axial e dos tratamentos de desbaste.

FIGURE 1: Variation of the average end split index of $\log$ (IMRT) of the Eucalyptus grandis dominant trees in function of the average relative position from axial direction and thinning treatments. 
Utilizando o modelo selecionado, os dados desse índice médio em função da posição relativa média no sentido base-topo foram então relacionados com as intervenções de desbaste e submetidos à análise de regressão com o emprego de variável Dummy (Tabela 3).

Os resultados obtidos indicam que a equação, selecionada no procedimento Stepwise de modelagem de regressão, assim como nas árvores dominantes, não pode ser utilizada para todos os desbastes analisados, pois a Dummy 1 (D1), associada ao tratamento testemunha sem desbaste, utilizada no modelo gigante, foi significativa em nível de $1 \%$ de probabilidade de erro. É interessante observar ainda, na Tabela 3, que as interações entre o desbaste executado e a posição relativa média no sentido base-topo apresentaram a mesma resposta das árvores dominantes, ou seja, não foram significativas ao mesmo nível de probabilidade já mencionado.
Dessa forma, para a equação com variáveis Dummy, o coeficiente de determinação ajustado $\left(\mathrm{R}^{2}{ }_{\mathrm{aj}}\right)$ apresentou um valor igual a 0,24 ; coeficiente de variação de $49 \%$ e erro padrão da estimativa igual a $0,58 \%$, mostrando-se significativa e sendo expressa por:

\section{IMRT $=\mathrm{e}$ $0,269138-0,000060 \mathrm{P}^{2}-0,365883 \mathrm{D} 1$}

Em que: $I M R T=$ índice médio de rachadura de topo de tora, $\% ; \mathrm{P}=$ posição relativa média no sentido base-topo, \%; D1 = variável Dummy correspondente ao tratamento testemunha sem desbaste.

Com o objetivo de verificar as diferenças do índice médio de rachadura de topo de tora existentes entre os desbastes executados, detectadas na análise de variância, os dados dessa característica foram plotados em função da posição relativa média no sentido axial, ajustando-se à equação selecionada

TABELA 3: Análise de variância da regressão com variável Dummy (tipo SS1) do índice médio de rachadura de topo de tora das árvores centrais de Eucalyptus grandis em função da posição relativa média e do tratamento de desbaste.

TABLE 3: Analysis of variance of regression with Dummy variable (SS1 type) of average end split index of logs of the Eucalyptus grandis central trees in function of the average relative position and thinning treatment.

\begin{tabular}{cccccc}
\hline FV & GL & SQ & QM & F & Prob. $>$ F \\
\hline Modelo & 13 & 6,805342 & 0,523488 & 2,09 & $0,0222^{*}$ \\
P2 & 1 & 2,717609 & 2,717609 & 10,85 & $0,0014^{* *}$ \\
D1 & 1 & 1,801904 & 1,801904 & 7,20 & $0,0087^{* *}$ \\
D2 & 1 & 0,026017 & 0,026017 & 0,10 & $0,7480^{\text {ns }}$ \\
D3 & 1 & 0,116781 & 0,116781 & 0,47 & $0,4965^{\text {ns }}$ \\
D4 & 1 & 0,014380 & 0,014380 & 0,06 & $0,8112^{\text {ns }}$ \\
D5 & 1 & 0,197868 & 0,197868 & 0,79 & $0,3765^{\text {ns }}$ \\
D6 & 1 & 0,454240 & 0,454240 & 1,81 & $0,1815^{\text {ns }}$ \\
D7 & 0 & 0 & 0 & 0 & 0 \\
D1.P2 & 1 & 0,593462 & 0,593462 & 2,37 & $0,1273^{\text {ns }}$ \\
D2.P & 1 & 0,012002 & 0,012002 & 0,05 & $0,8272^{\text {ns }}$ \\
D3.P2 & 1 & 0,030939 & 0,030939 & 0,12 & $0,7261^{\text {ns }}$ \\
D4.P2 & 1 & 0,430871 & 0,430871 & 1,72 & $0,1930^{\text {ns }}$ \\
D5.P2 & 1 & 0,221437 & 0,221437 & 0,88 & $0,3496^{\text {ns }}$ \\
D6.P2 & 1 & 0,187828 & 0,187828 & 0,75 & $0,3888^{\text {ns }}$ \\
D7.P2 & 0 & 0 & 0 & 0 & 0 \\
Erro & 89 & 22,288554 & 0,250433 & - & - \\
Total & 102 & 29,093896 & - & - & - \\
\hline
\end{tabular}

Em que: $\mathrm{FV}=$ fonte de variação; $\mathrm{GL}=$ graus de liberdade; $\mathrm{SQ}=$ soma dos quadrados; $\mathrm{QM}=$ quadrado médio; $\mathrm{F}=$ valor de $\mathrm{F}$ calculado; Prob. $>\mathrm{F}$ = nível de probabilidade de erro; $\mathrm{P}=$ posição relativa média no sentido base-topo, \%; $\mathrm{D}_{, i,}=$ tratamentos de desbaste (Dummy); $\mathrm{D}_{, \mathrm{i}}, \mathrm{P}^{2}=$ interação tratamento (Dummy) com a variável $\mathrm{P}^{2} ; * *=$ significativo ao nível de $1 \%$ de probabilidade de erro; $*$ = significativo ao nível de $5 \%$ de probabilidade de erro; ns = não significativo ao nível de $5 \%$ de probabilidade de erro. 
pelo procedimento Stepwise. Os valores desse índice, bem como sua respectiva variação, nas árvores centrais de Eucalyptus grandis, estimados para as
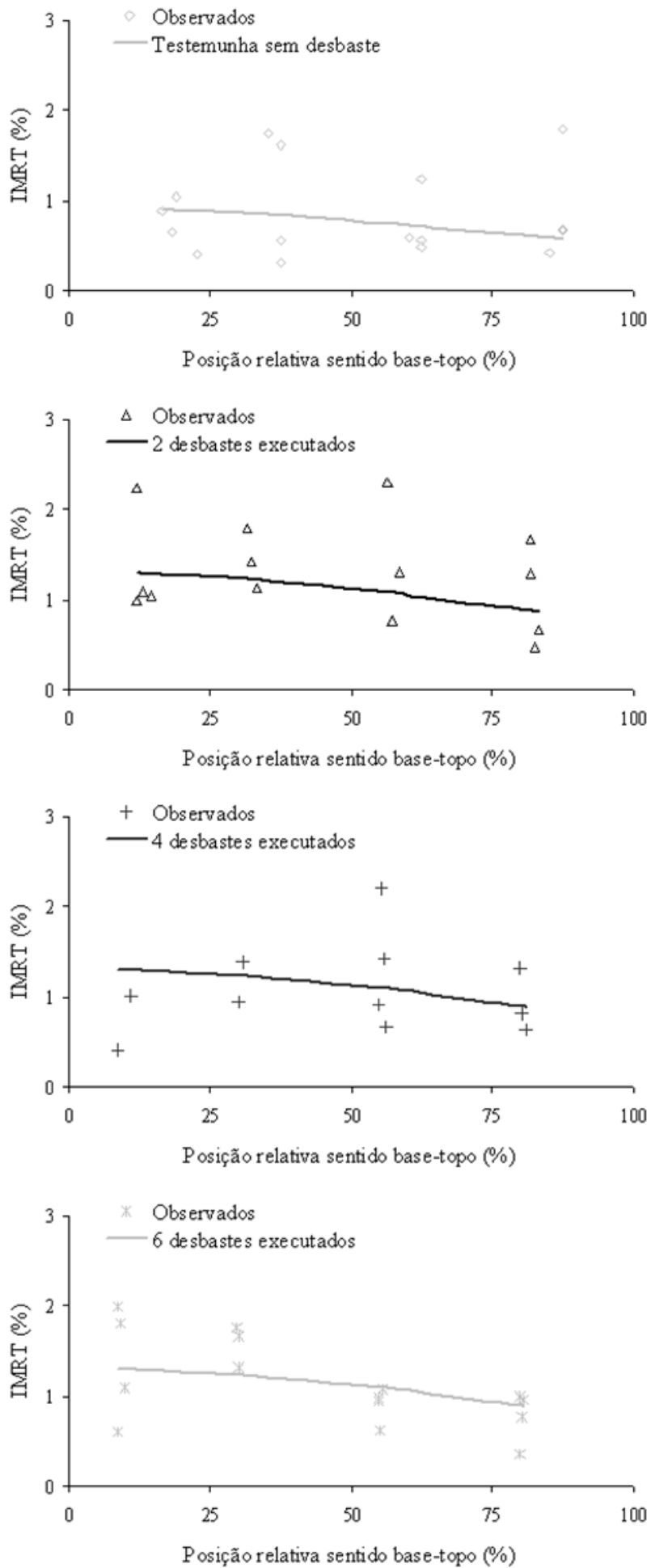

sete intervenções silviculturais de desbaste pelo modelo gigante já mencionado, podem ser observados na Figura 2.

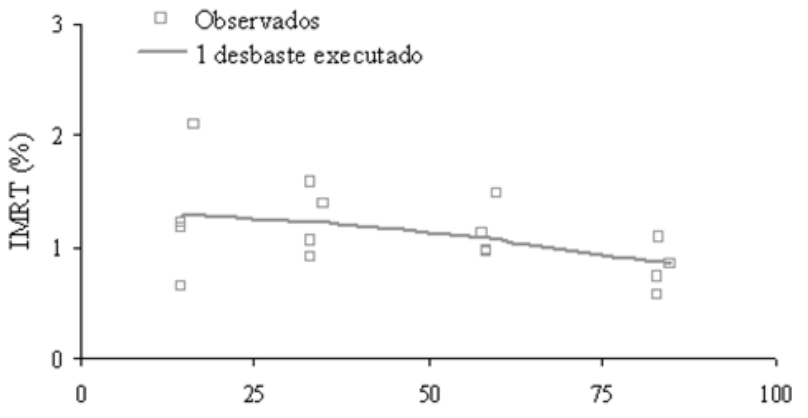

Posição relativa sentido base-topo (\%)

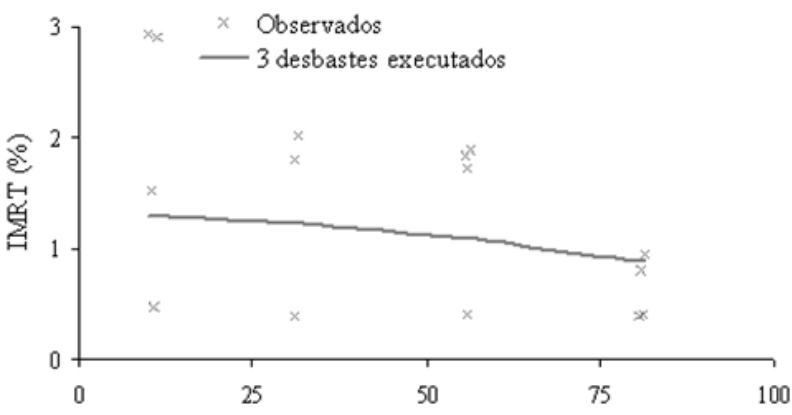

Posição relativa sentido base-topo (\%)
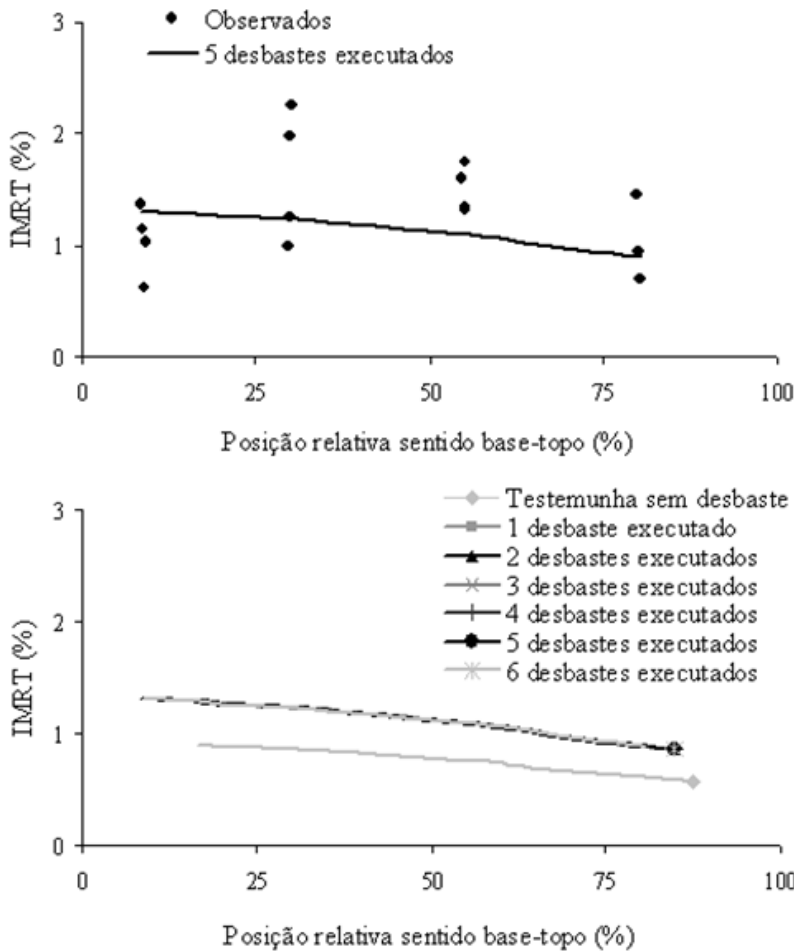

FIGURA 2: Variação do índice médio de rachadura de topo de tora (IMRT) das árvores centrais de Eucalyptus grandis em função da posição relativa média no sentido axial e dos tratamentos de desbaste.

FIGURE 2: Variation of the average end split index of $\log$ (IMRT) of the Eucalyptus grandis central trees in function of the average relative position from axial direction and thinning treatments. 
Analisando a Figura 2, verifica-se que o padrão de variação longitudinal do índice médio de rachadura de topo de tora, nas árvores centrais da madeira de Eucalyptus grandis, não apresentou a mesma tendência que nas dominantes, ou seja, para esse estrato, ocorreu um decréscimo desse índice no sentido base-topo. Os resultados encontrados corroboram o modelo de variação longitudinal decrescente descrito por Chafe (1985) e Schacht e Garcia (1997).

Entretanto, cabe ressaltar que a variação decrescente foi semelhante em todos os desbastes estudados, evidenciando que essa intervenção silvicultural, assim como nas árvores dominantes, também não altera o comportamento do índice de rachadura de topo de tora no sentido axial.

Com relação ao efeito dos desbastes, pode-se observar, na Figura 2, que houve influência das diferentes formas de competição no índice médio de rachadura de topo de tora. Entretanto, diferentemente da variação encontrada nas árvores dominantes, as árvores centrais apresentaram uma tendência positiva entre o índice de rachadura e o desbaste aplicado.

Contudo, nota-se que essa relação é verdadeira até o tratamento 2, correspondente a um desbaste executado, pois intensidades superiores não influenciaram significativamente o desenvolvimento do índice médio de rachadura de topo de tora.

\section{Árvores dominantes e centrais}

A hipótese de homogeneidade de variâncias, realizada pelo Teste de Bartlett, entre as médias estimadas pelas equações selecionadas para descrever o índice médio de rachadura de topo de tora e, também, para as médias observadas desse índice, nas árvores dominantes e centrais, foi aceita em nível de $1 \%$ de probabilidade de erro.

Dessa forma, o Teste-t para duas amostras com variância equivalentes foi aplicado, resultando na rejeição da hipótese de igualdade de variação axial entre os estratos, tanto para os valores médios observados do índice médio de rachadura de topo de tora ( $\mathrm{t}$ cal $=3,446>\mathrm{t}$ tab $=1,972)$ quanto para os estimados pelas equações em árvores dominantes e centrais ( $\mathrm{t}$ cal $=6,226>\mathrm{t}$ tab $=1,972$ ).

Assim, os resultados obtidos indicam que essa característica apresenta uma variação axial heterogênea entre os estratos estudados, evidenciando que os dados do índice médio de rachadura de topo de tora das árvores dominantes e centrais não podem ser agrupados para analisar essa tendência.

\section{CONCLUSÕES}

Os resultados obtidos no estudo, para a espécie Eucalyptus grandis, aos 18 anos, permitem concluir que:

O padrão de variação do índice médio de rachadura de topo das toras no sentido longitudinal não apresentou a mesma tendência nos estratos analisados, sendo que, nas árvores dominantes, esse índice aumentou da base até, aproximadamente, $30 \%$ da altura comercial, seguido de decréscimo com a altura. Com relação às árvores centrais, ocorreu um decréscimo desse parâmetro no sentido base-topo.

Os diferentes tratamentos de desbaste não modificaram o padrão longitudinal descrito para o índice médio de rachadura de topo das toras das árvores dominantes e centrais.

O desbaste induz à variação do índice médio de rachadura de topo das toras, tanto nas árvores dominantes como nas centrais, porém, sem apresentar uma tendência sistemática.

\section{AGRADECIMENTOS}

Os autores agradecem o apoio da Empresa Flosul Indústria e Comércio de Madeiras Ltda., pela colaboração para a realização deste trabalho.

\section{REFERÊNCIAS BIBLIOGRÁFICAS}

ASSIS, T. F. Qualificação tecnológica da madeira de Eucalyptus para serraria: Aspectos genéticos e de manejo. In: SIMPÓSIO LATINO-AMERICANO SOBRE MANEJO FLORESTAL, 1., 2000. Santa Maria, UFSM, Anais... Santa Maria: Programa de Pós-graduação em Engenharia Florestal , 2000. p. 59-80.

BARISKA, M. A method for predicting timber quality in eucalypts. In: VON GADOW, K.; BREDENKAMP, B. V. Management of Eucalyptus grandis in South Africa. Stellenbosch: Southern African Institute of Forestry, 1990. p.215-228.

BREDENKAMP, B. The CCT concept in spacing research - a review. In: THE IUFRO SYNPOSIUM. 1984, Pietermaritzburg. Proceedings... Pietermaritzburg: IUFRO, 1984.

CALONEGO, W. F.; SEVERO, D. T. E. Efeito da vaporização de toras na redução dos defeitos associados a tensão de crescimento de Eucalyptus grandis, Ciência Florestal, n. 4, v. 15, p. 431-440, out./dez. 2005.

CHAFE, S. C. Variation in longitudinal growth 
stress, basic density and modulus of elasticity with height in the tree. Australian Forest Research, Canberra, v. 11, n.1, p. 79-82. 1981.

CHAFE, S. C. Variation in longitudinal growth stress with height in trees of Eucalyptus nitens Maiden. Australian Forest Research, Canberra, v. 15, n.1, p. 51-55. 1985.

DEL MENEZZI, C. H. Utilização de um método combinado de desdobro e secagem para a produção de madeira serrada de Eucalyptus grandis Hill ex Maiden e Eucalyptus cloeziana F. Muell. 1999. 77 f. Dissertação (Mestrado em Ciências Florestais)-Escola Superior de Agricultura "Luiz de Queiroz", Piracicaba, 1999.

FERRAND, J. C. Growth stresses and silviculture of eucalypts. Australian Forest Research, Canberra, v. 13, n. 1, p. 75-81. 1983.

GARCIA, J. N. Técnicas de desdobro de eucalipto. In: SEMINÁRIO INTERNACIONAL DE UTILIZAÇÃO DE EUCALIPTO PARA SERRARIA, 1., 1995, Piracicaba. Anais... Piracicaba: IPEF, 1995, p.59-67.

GONÇALEZ, J. C. et al. Características tecnológicas da madeira de Eucalyptus grandis W.Hill ex Maiden e E. Cloeziana F.Muell visando ao seu aproveitamento na indústria moveleira. Ciência Florestal, Santa Maria, v. 16, n. 3, p. 329-341, jul./ out. 2006.

HASELEIN, C. R. et al. Características tecnológicas da madeira de árvores matrizes de Eucalyptus grandis. Ciência Florestal, Santa Maria, v. 14, n. 2, p. 145-155, abr.jun. 2004.

HILLIS, W. E. Chemical aspects of heartwood formation. Wood Science and Technology, Madison, v. 2, n. 4, p. 241-259, Dec. 1968.

JANKOWSKY, I. P. Equipamentos e processos para a secagem de madeiras. In: SEMINÁRIO INTERNACIONAL DE UTILIZAÇÃO DA MADEIRA DE EUCALIPTO PARA SERRARIA, 1995, São Paulo. Anais... São Paulo: IPEF/IPT/IUFRO/LCF/ESALQ/USP, 1995, p. 109-118.

KUBLER, H. Growth stresses in trees and related wood properties. Forestry Abstracts, Oxford, v. 48, n.3, p. 131-189, 1987.

LATORRACA, J. V. F.; ALBUQUERQUE, C. E. C. Efeito do rápido crescimento sobre as propriedades da madeira. Floresta e Ambiente, Seropédica, v. 7, n. 1, p. 279-291. jan./dez. 2000.

LIMA, I. L. Variação de propriedades indicativas da tensão de crescimento em função da posição na árvore e da intensidade de desbaste. 2000.90 f. Dissertação (Mestrado em Engenharia Florestal)Escola Superior de Agronomia "Luiz de Queiroz", Universidade de São Paulo, Piracicaba, 2000.

LIMA, I. L.; GARCIA, J. N.; NOGUEIRA, M. C. S. Influência do desbaste nas tensões de crescimento de Eucalyptus grandis Hill ex Maiden. Scientia Forestalis, Piracicaba, v. 58, p. 111-125, dez. 2000. LIMA, I. L.; GARCIA, J. N.; STAPE, J. L. Influência do desbaste e da fertilização no deslocamento da medula e rachaduras de extremidade de tora de Eucalyptus grandis Hill ex Maiden. Cerne, Lavras, v. 13, n. 2, p. 170-177, abr./jun. 2007.

MALAN, F. S. Relationships between growth stress and some tree characteristics in South African grown Eucalyptus grandis. South African Forestry Journal, Pretoria, n. 144, p. 43-46. 1988.

MALAN, F. S. The control of end splitting in sawlogs: a short literature review. South African Forestry Journal, Pretoria, n. 109, p.14-79, 1979.

MELlADO, E. C. E. R. Contribuição ao desenvolvimento tecnológico para a utilização de madeira serrada de Eucalyptus grandis Hill ex Maiden na geração de produtos com maior valor agregado. 1993. 133 f. Dissertação (Mestrado em Engenharia Florestal)-Universidade Federal do Paraná, Curitiba, 1993.

MIRANDA, J. A. C.; NAHUZ, M. A. R. Estudo do espaçamento de plantio de Eucalyptus saligna Smith nos índices de rachamento após o desdobro e após a secagem. Scientia Forestalis, Piracicaba, n. 55, p. 107-116, jun. 1999.

NUTTO, L.; MAESTRI, R. Growth rate and growth stresses in brazilian eucalypts: preliminary results of growth and quality modeling on an individual tree basis. In: IUFRO DIVISION 5.01.04 BIOLOGICAL IMPROVEMENT OF WOOD PROPERTIES. Proceedings... Harrison Hot Springs, 2002.

OLIVEIRA, J. T. S. et al. Caracterização da madeira de sete espécies de eucaliptos para a construção civil. Scientia Forestalis, Piracicaba, n. 56, p. 113124, dez. 1999.

PURNELL, R. C. Variation in wood properties of Eucalyptus nitens in a provenance trial on the eastern Transvaal highveld in South Africa. South African Forestry Journal, Pretoria, n. 144, p. 1022, 1988.

REINSTORF, L. O. The influence of stand density on growth of Pinus elliottii. 1970. $62 \mathrm{f}$. Dissertação (Mestrado)-University of Stellenbosch, Stellenbosch, 1970.

SANTOS, P. E. T. Avaliação de características 
tecnológicas de madeira para serraria em progênies de polinização aberta de eucalipto e implicações para o melhoramento genético. 2002. 153 f. Tese (Doutorado em Agronomia)Escola Superior de Agronomia "Luiz de Queiroz", Universidade de São Paulo, Piracicaba, 2002.

SAS - STATISTICAL ANALYSIS SYSTEM: Programa de computador, ambiente VM. Cary, 1993. Versão 6.08.

SCHACHT, L.; GARCIA, J. N. Variação entre e intra clones nas rachaduras de topo em Eucalyptus urophylla. In: CONFERÊNCIA IUFRO SOBRE SILVICULTURA E MELHORAMENTO DE EUCALIPTOS, 1997, Salvador. Anais... Colombo: EMBRAPA/CNPF, 1997. p.401-404.

SCHACHT, L.; GARCIA, J. N.; VENCOVSKY, R. Variação genética de indicadores de tensão de crescimento em clones de Eucalyptus urophylla. Scientia Forestalis, Piracicaba v. 54, p. 55-68, dez. 1998.

TREVISAN, R. Efeito do desbaste nos parâmetros dendrométricos e na qualidade da madeira de
Eucalyptus grandis W. Hill ex Maiden. 2010. 174 f. Tese (Doutorado em Ciências Florestais) Universidade Federal de Santa Maria, Santa Maria, 2010.

TREVISAN, R. et al. Variação radial da massa específica básica da madeira de Eucalyptus grandis W. Hill ex Maiden. Floresta, Curitiba, v. 38, n. 3, p. 553-559, jul./set. 2008.

TRUGILHO, P. F. et al. Avaliação da tensão de crescimento em clones de Eucalyptus. Floresta e Ambiente, Seropédica, v. 9, n. 1, p. 38-44, jan./dez. 2002.

TRUGILHO, P. F. et al. Efeitos da idade e classe diamétrica na deformação residual longitudinal em árvores de Eucalyptus dunnii Maiden. Revista Árvore, Viçosa, v. 28, n. 5, p. 725-731, set./out. 2004.

WILKINS, A. P.; KITAHARA, R. Silvicultural treatments and associated growth rates, growth strains and wood properties in 12,5-year-old Eucalyptus grandis. Australian Forestry, Yarralumla, v. 54, n. 1/2, p.99-104, 1991. 